\title{
REGRESAR PARA CONSTRUIR: PRÁCTICAS FUNERARIAS E IDEOLOGÍA(S) DURANTE LA OCUPACIÓN INKA EN CUTIMBO, PUNO-PERÚ
}

\author{
RETURNING TO BUILD: FUNERARY PRACTICES AND IDEOLOGY (IES) \\ DURING THE INKA OCCUPATION OF CUTIMBO, PUNO-PERU
}

\author{
Henry Tantaleán ${ }^{1}$
}

\begin{abstract}
Este artículo describe nuestras investigaciones arqueológicas realizadas en el sitio prehispánico de Cutimbo (Puno-Perú), el mismo que fue ocupado durante el período Altiplano (1.100-1.470 d.C.) y reocupado durante la época inka (1.470-1.532 d.C.). Nuestras excavaciones se concentraron en las torres funerarias (chullpas) monumentales y sus áreas asociadas, ofreciendo evidencia de la existencia de prácticas sociales que reprodujeron asimetrías económicas, políticas e ideológicas entre Inkas y lupakas. Dicha asimetría social no sólo se dio entre la sociedad dominada (Lupaka) y la dominante (Inka), sino también en el seno de la misma sociedad Lupaka, un proceso histórico que trascendió a la ocupación inka de la zona.
\end{abstract}

Palabras claves: asimetría social, coerción, ideología, lupakas, chullpas.

This paper describes archaeological research carried out in Cutimbo (Puno-Peru), a prehispanic settlement occupied during the Altiplano Period (1,100-1,470 A.D.) and re-occuppied durig the Inka epoch (1,470-1,532 A.D.). Our excavations focus on the monumental funerary towers (chullpas) and related areas which offered evidence consistent with the existence of economic, political and ideological asymmetries between Inkas and lupakas. This social asymmetry not only existed between dominant society (Inka) and dominated society (Lupaka) but also within Lupaka society, a historical process trascending the Inka occupation of the area.

Key words: Social asymmetry, coercion, ideology, lupakas, chullpas.

Los lupakas fueron una de las sociedades más complejas y poderosas asentadas en torno al lago Titicaca a la llegada de los ejércitos del Estado Inka alrededor de 1.470 d.C. (Stanish 2003:237). Mediante las fuentes etnohistóricas se ha tratado de recuperar los modos de vida de las sociedades altiplánicas como la lupaka, pues ésta se ha detallado en muchas crónicas tempranas (Diez de San Miguel 1964 [1567]; Gutiérrez Flores 1964 [1574]; Murra 1968). A consecuencia de este conocimiento mediante fuentes históricas, el trabajo de contrastación arqueológica en muchos casos ha sido desplazado (Malpass 1993:2). Sin embargo, toda una nueva generación de arqueólogos y arqueólogas han comenzado a establecer líneas de investigación que están empezando a llenar ese vacío en las representaciones de las sociedades altiplánicas prehispánicas (p. ej. Stanish et al. 2005).

Nosotros tuvimos la oportunidad de trabajar en el altiplano andino, específicamente en el De- partamento de Puno, Perú, recorriendo e investigando sitios prehispánicos de diferentes épocas. De entre ellos, tuvimos a cargo la ejecución de los trabajos arqueológicos en Cutimbo, uno de los sitios arqueológicos más representativos del área lupaka $y$ al que se le hace continua referencia sin que este haya sido investigado sistemáticamente (p. ej. Frye y de la Vega 2005; Hyslop 1977b, 1990, Isbell 1997; Stanish 2003).

En este artículo se enfoca el tema de la lucha por la supervivencia real e ideológica de la elite lupaka representada en los restos de su materialidad social, en este caso en el sitio de Cutimbo. Para ello partiremos de la premisa que las estrategias inka de dominación imperial estuvieron en correspondencia con las prácticas socioparentales, socioeconómicas y sociopolíticas de las sociedades a las que se enfrentaron ${ }^{1}$. Así pues, de acuerdo con las características infraestructurales de la sociedad a dominar se ejercía un tipo u otro de control de la

\footnotetext{
1 Departamento de Prehistoria, Universidad Autónoma de Barcelona, España, y Universidad Nacional Mayor de San Marcos, Lima, Perú. henrytantalean@yahoo.es
} 
producción de la vida social de la población dominada, lo que consecuentemente también supondría una confrontación entre la ideología dominante y la ideología dominada. Para comprobar dicho supuesto en primera instancia utilizaremos la información disponible para el área que nos ocupa y la contrastaremos con los hallazgos realizados en nuestra investigación.

\section{Dominación del Estado Inka y Reestructura- ción de la Sociedad Lupaka}

El registro arqueológico regional puede ayudarnos a entender de mejor manera los cambios producidos posteriores a la ocupación de un territorio por un grupo social dominante y que en el caso de la llegada de los inkas al área lupaka parece ser dramático. Originalmente, el patrón de asentamiento lupaka (1.100-1.470 d.C.) se realizó en dos niveles principalmente: (a) pueblos fortificados en cumbres de cerros (pukaras) o mesetas, donde las viviendas presentan una nucleación de estructuras de planta circular con techos de falsa bóveda y (b) aldeas dispersas reconocidas como concentraciones de estructuras domésticas y/o dispersión de cerámica en las áreas llanas (Frye y de la Vega 2005; Hyslop 1976, 1977a, 1977b, 1978; Stanish 2003; Stanish et al. 1997). Sin embargo, una vez dominadas dichas sociedades por los inkas, un nuevo patrón de asentamiento fue impulsado por sus dirigentes, desplazando y concentrando a la población local en asentamientos ubicados en el área cercana a las orillas del lago Titicaca y articulados mediante el camino inka principal (Arkush 2005; Hyslop 1977b, 1984; Stanish 1997) con el objetivo principal de conseguir un mayor control de la población y su producción ${ }^{2}$. En el caso de la producción material, por ejemplo, la tradición cerámica local se mezcló con la de los dominantes en los talleres, para dar paso a dos de los estilos Inka-asociados más importantes del lago Titicaca: el Sillustani Policromo y el Inka-Chucuito (Tschopik 1946).

Como Stanish (2000) ha argumentado, previamente a la ocupación inka, la sociedad lupaka estaba cercana a una organización de tipo estatal, posición que permitiría a la elite conservar gran parte de sus privilegios socioeconómicos e incluso mantener su jerarquía política local adentro de la jerarquía política inka. Estudios de patrones de asentamiento (Frye 1997; Frye y de la Vega 2005;
Stanish et al. 1997) también evidencian una complejidad socioeconómica que incluía la localización de asentamientos relacionados con la explotación de los recursos ganaderos durante el período preinka Altiplano y un ambiente de precariedad política (materializado en los pukaras o fortalezas naturales como la de Cutimbo) como producto de los enfrentamientos entre grupos de interés dentro de la sociedad o fuera de ella (ver también Hyslop 1977b). Finalmente, según la evidencia etnohistórica existiría un ejército lupaka (Cieza de León 1929 [1553]; Julien 1983) que permitiría el control de la población y el resguardo de los intereses materiales de la elite lupaka.

Asumiendo que las asimetrías sociales también se pueden materializar en las prácticas funerarias y que en ellas se reflejaría la ideología del grupo de interés que las realizó (Lull 2000) y que promovería la reproducción de la jerarquía social (distancia social) del "mundo de los vivos" o de la producción de la vida social (Castro et al. 1998), las prácticas funerarias constatadas en diferentes investigaciones en el área lupaka harían patente un acceso diferencial a lugares de entierro, consumo pasivo de la producción o amortización de bienes, lo que plantea que la sociedad lupaka antes y durante la ocupación inka estaba dividida en diferentes grupos socioeconómicos ${ }^{3}$.

\section{Prácticas Funerarias de la Sociedad Lupaka}

En el área ocupada por los lupaka son representativas las formas de enterramiento utilizadas. Se han hallado hasta cuatro tipos de ellas: torres funerarias o chullpas, cistas de paredes de piedra, las "slab-cist tombs" y cuevas funerarias (Tschopik 1946; Stanish 2003:93-95, 229-331). En el caso de los tres primeros tipos de estructuras funerarias, estas pueden ser construidas y concentradas preferentemente en lugares que sobresalen en el espacio geográfico como mesetas, colinas, montículos pero también se encuentran dispersas entre las zonas llanas o en los campos de cultivo (Stanish 1994, 2003).

De todas las estructuras funerarias, las chullpas son las más conocidas y serían una forma de arquitectura funeraria local y que subsiste con mejoras técnicas durante la ocupación inka. Las chullpas del altiplano, en especial las monumentales, han sido objeto de numerosas descripciones desde épocas muy tempranas de contacto con 
Europa (siglo XVI) hasta la actualidad (p. ej. Ayca 1995; Bandelier 1905; Cieza 1929 [1553]; Cobo 1964 [1653]; Guamán Poma 1987 [1615]; Gil García 2000, 2001; Hyslop 1977a; Isbell 1997; Lumbreras 1974; Nordenskiöld 1953; Squier 1974 [1877]; Tschopik 1946; Vásquez 1937, 1939).

Las chullpas están construidas con piedra aunque hay algunas realizadas en barro (Gisbert et al. 1996; Isbell 1997:172-173; Kesseli 2001; Kesseli et al. 1999; Stanish 2003:231). Por lo general, las chullpas tienen un pequeño acceso hacia la cámara funeraria orientado casi siempre hacia al este. Estas tumbas monumentales serían utilizadas como referentes o hitos en el espacio geográfico construyendo un paisaje social en el que se desarrolla la vida de la población local y, en este caso, perseguirían perennizar el poder de la clase dominante aun después de la muerte. En este caso concreto, dichos accidentes geográficos han sido modificados por los dirigentes de la sociedad, inscribiendo sus estructuras arquitectónicas en el paisaje natural como muestra de un dominio de la realidad social y de un manejo racional de su espacio sagrado. Por ello, no es gratuito que se escojan las mesetas para su construcción pues muchas de ellas son reconocidas como deidades protectoras (Apus). De hecho, el concepto de un lugar de origen mitológico ancestral para cada pueblo (Paqarina) está bastante arraigado en los Andes, y se reconocen en los mitos que señalan lugares (casi siempre accidentes geográficos) de donde proceden los primeros seres humanos (Arkush 2005:240) y lugar a los que deben regresar al morir. De esta ideología provendría la primaria sacralización de los accidentes geográficos, como sería el caso de Cutimbo.

La segunda forma de enterramiento más extendida en el área lupaka es la cista subterránea y aunque pueden estar aisladas suelen conformar concentraciones y en algunos casos pueden estar asociadas a torres funerarias. Dichas cistas, de paredes de piedras con base y cobertura realizadas con lajas del mismo material, contienen por lo general a un solo individuo e incluye un ajuar funerario de los cuales han sobrevivido, por las condiciones climáticas del altiplano, solamente los objetos más resistentes como las vasijas cerámicas y los objetos metálicos como los tupus.

La tercera forma de enterramiento es la "slabcist tomb", la cual no ha sido hallada en nuestras investigaciones en Cutimbo y hasta la fecha aparte de algunas referencias de su existencia durante prospecciones arqueológicas (Stanish et al. 1997; Stanish 2003:229-230) sólo ha sido descrita detalladamente por Tschopik (1946) y se caracterizarían principalmente por ser tumbas colectivas.

Finalmente están las cuevas funerarias, en las cuales se realizaron varios eventos de internamiento de cadáveres y fueron selladas al completarse su capacidad. El mejor caso estudiado es el de la cueva Molino-Chilacachi (de la Vega et al. 2005), gracias al cual ahora sabemos que en dichas cuevas los difuntos se depositaban en posición fetal envueltos individualmente en tejidos de fibra de totora y acompañados de vasijas cerámica y otros artefactos. Cuevas similares aunque no tan profundas y muy disturbadas han sido halladas en Cutimbo. Dichas cuevas se encuentran en los farallones cercanos a la cima de la meseta y, aunque muchas de ellas se hallaban disturbadas, se reconoció un gran número huesos humanos asociados a fragmentos de cerámica Inka-Chucuito. Las paredes de algunas cuevas incluyeron paneles con arte rupestre con diseños inka hallados en la cerámica recuperada en nuestras excavaciones (Tantaleán y Pérez 2000).

En nuestras investigaciones en Cutimbo hallamos tres de las formas de enterramiento descritas arriba, que conformando una unidad espacial y temporal nos ofrecen una idea del conjunto de prácticas funerarias que se realizaron en un período de tiempo corto (ocupación inka del altiplano).

\section{Los Trabajos Arqueológicos en Cutimbo}

Cutimbo se encuentra localizado al sureste de la ciudad de Puno (Figura 1). Se trata de una gran meseta que se levanta sobre el relieve regularmente llano de la zona. Ha sido señalado por Hyslop (1976, 1977b, 1990:278) como la capital política de la sociedad lupaka, básicamente por la existencia de un gran número de "bases de casas" y torres funerarias (Figura 2). De hecho, Frye y de la Vega (2005) señalan a Cutimbo como uno de los sitios más grandes dentro de su área de prospección. Sin embargo, habría que anotar que una "capital" debería encontrarse en la zona nuclear de un territorio y no en un extremo tan cercano a la "frontera" con los Colla ${ }^{4}$. Más plausible sería lo que plantea Frye (1997), que Cutimbo sería el asentamiento principal de uno de los grupos sociales que conformarían el área de una confederación política previa a la ocupación definitiva del territorio por los inka. 


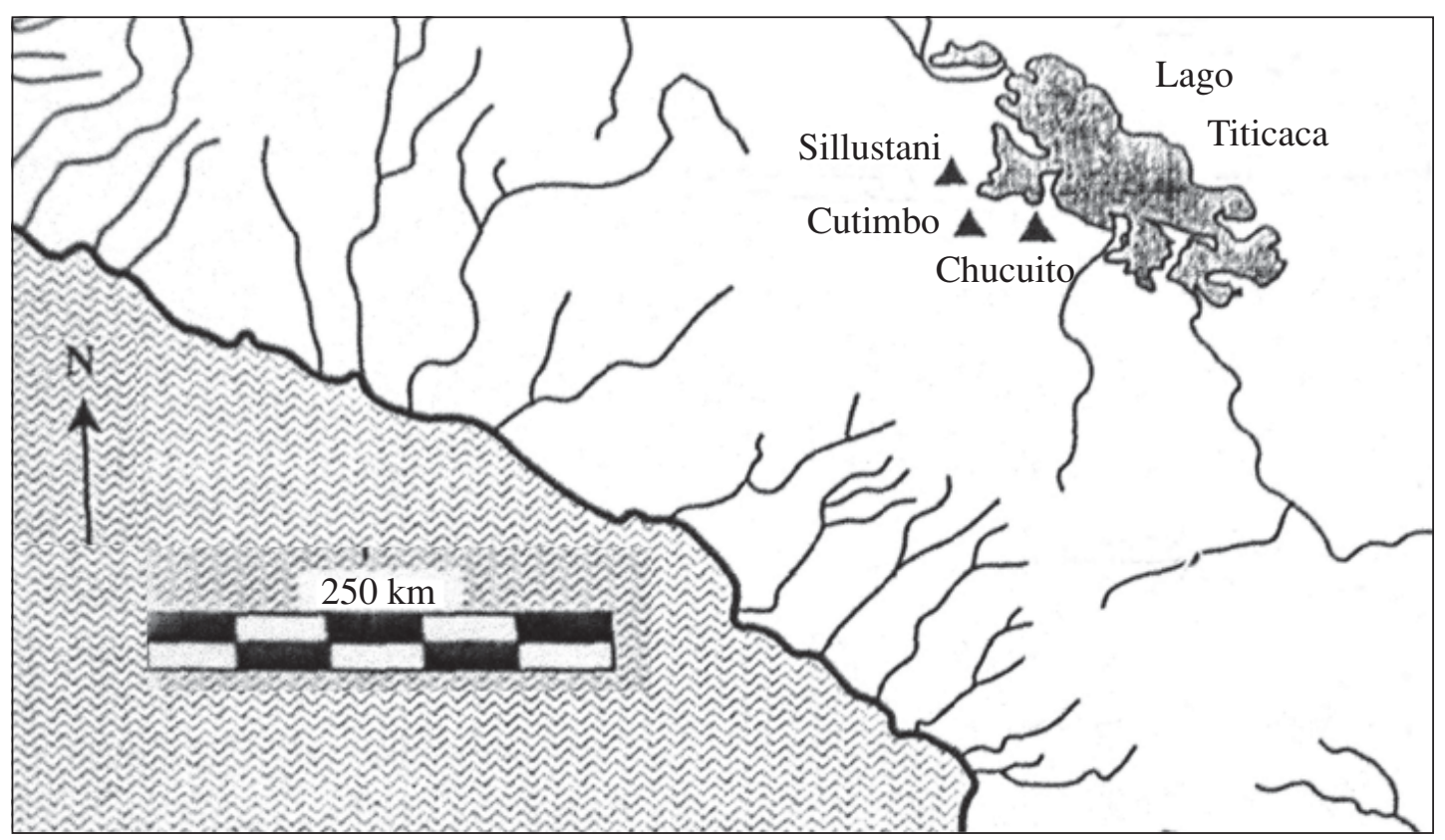

Figura 1. Ubicación de Cutimbo (realizado a partir de Isbell 1997: Mapa 6.1).

Location of Cutimbo (after Isbell 1997: Map. 6.1).

Cutimbo es un sitio arqueológico conocido por la mayoría de los investigadores que han trabajado extensivamente en esta área (Hyslop 1977, 1979; Tschopik 1946; Vázquez 1939) y aunque parece tener un valor muy importante para diversas representaciones acerca de la naturaleza de la sociedad lupaka (p. ej. Frye 1997, 2005; Frye y de la Vega 2005; Gil García 2000; Hyslop 1976, 1990; Isbell 1997; Stanish 2003) nunca se había excavado sistemáticamente en él y las asociaciones cronológicas y sociales habían sido realizadas simplemente por características formales arquitectónicas y por las referencias etnohistóricas. Se ha dicho que las estructuras funerarias chullparias se construyen desde el período Altiplano (1.100 -1.450 años d.C.) hasta el fin de la época inka a la llegada de los españoles alrededor de 1.532 d.C. (Hyslop 1977b; Stanish 2003:212). Sin embargo, nadie había excavado en el sitio, por lo que todo quedaba bastante inconsistente en relación con la época de construcción de las estructuras funerarias, principalmente de las chullpas monumentales que forman el núcleo de nuestra investigación.

Asimismo, Cutimbo es uno de muchos complejos funerarios en la cuenca oeste del Lago Titicaca como Sillustani (Ayca 1995), Tanka Tanka (Hyslop 1977b) o Acora (Hyslop 1990:249) que conformarían un patrón que consistiría en la concentración de estructuras funerarias monumentales sobre mesetas o accidentes geográficos que se elevan sobre la superficie terrestre. En el caso concreto de Cutimbo, aparte de las cuestiones relacionadas con la ideología, estuvo principalmente orientado a la explotación extensiva de rebaños de camélidos y, de hecho, su localización altitudinal favorece dicha actividad económica (Frye y de la Vega 2005; Stanish 1994). Adicionalmente, el arte rupestre de las cuevas funerarias representa con mucha recurrencia esta actividad (Tantaleán y Pérez 2000). También se puede apreciar que la mayor parte de la cima de la meseta (unas 25 hectáreas según Hyslop (1977b:225)) estuvo ocupada por estructuras circulares que podrían ser viviendas (ver plano de la meseta en Frye y de la Vega 2005: Figura 11.5), aunque excavaciones de algunas de ellas realizadas por Edmundo de la Vega (Frye y de la Vega 2005) y las de Eduardo Arizaca durante nuestra estadía no revelaron ocupación humana, lo que plantearía que algunas serían utilizadas como depósitos. Lo que sí parece seguro es que el sitio comenzó a utilizarse en el período Altiplano, pues, según un fechado por AMS, este fue ocupado tan temprano como 1.320 d.C. (Frye y de la Vega 2005:178). 


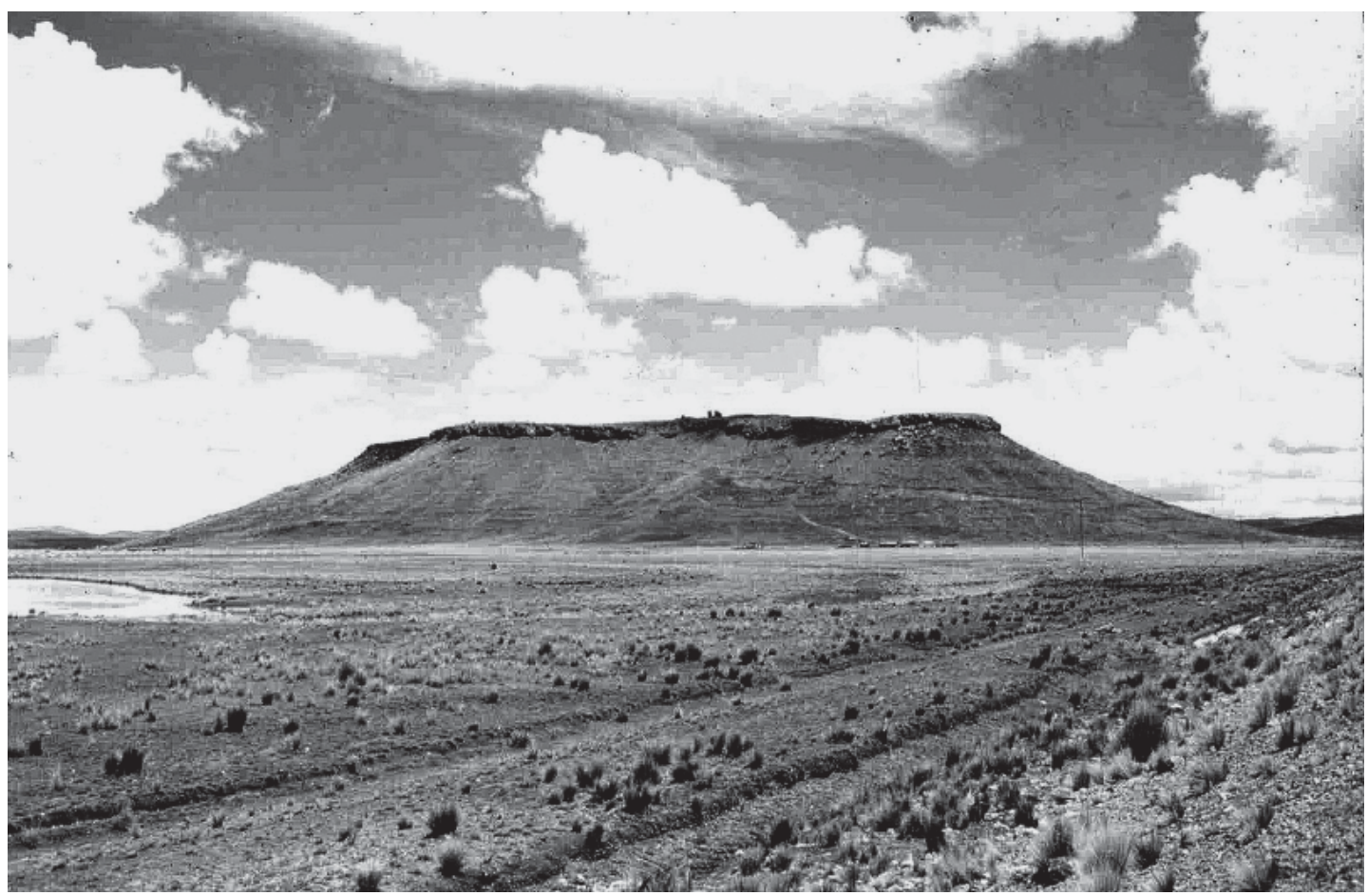

Figura 2. La meseta de Cutimbo. En la parte superior se encuentra la mayor acumulación de estructuras domésticas y funerarias. The Cutimbo plateau. The largest accumulation of domestic and funerary structurs is on the top.

Los resultados de nuestras investigaciones se presentan de manera sintetizada y los hemos dividido de acuerdo a las estructuras funerarias intervenidas $^{5}$. Nuestro objetivo particular fue definir si las chullpas monumentales incluían en sus alrededores áreas de actividad social prehispánica y, asimismo, si las mismas concentraban estructuras funerarias no obvias en superficie (como cistas funerarias) como las que habíamos observado en Sillustani y que revelarían, además, que las cistas se seguían construyendo como forma de enterramiento durante la ocupación inka del área lupaka. Asimismo, mediante nuestras investigaciones pudimos comprobar que todas las torres funerarias habían sido disturbadas con anterioridad, una situación que se observa en tumbas de este tipo dada su monumentalidad y los ajuares funerarios que seguramente contenían y que ya describían los primeros cronistas castellanos.

\section{Las Torres Funerarias o Chullpas}

Excavamos tres chullpas funerarias en área, tanto interior como exteriormente. La primera de ellas, de medianas dimensiones (chullpa 20), está ubicada en la ladera norte de la meseta (Figuras 3 y 4). Tiene planta cuadrangular y la pendiente sobre la que ésta se construyó fue nivelada mediante tres terrazas consecutivas y una plataforma cuadrangular alrededor de la base de la chullpa. Posee cuatro hornacinas trapezoidales en las paredes interiores de la cámara funeraria donde estarían colocados los cadáveres. Un segundo piso cumpliría el mismo fin y que se infiere por bloques líticos que sobresalen de la pared y que habrían soportado una estructura de material perecedero, probablemente madera. Exteriormente algunos bloques líticos están decorados con representaciones en alto relieve de serpientes y su acceso está orientado hacia el este.

Las otras dos chullpas intervenidas (chullpas 1 y 2) son las más importantes del sitio, tanto por su monumentalidad como por su emplazamiento en la parte superior de la meseta (Figura 5). La chullpa 2 posee planta circular y una mampostería externa "almohadillada" del tipo poligonal ciclópeo, realizada con bloques de andesita, extraída de una cantera localizada en la misma meseta 


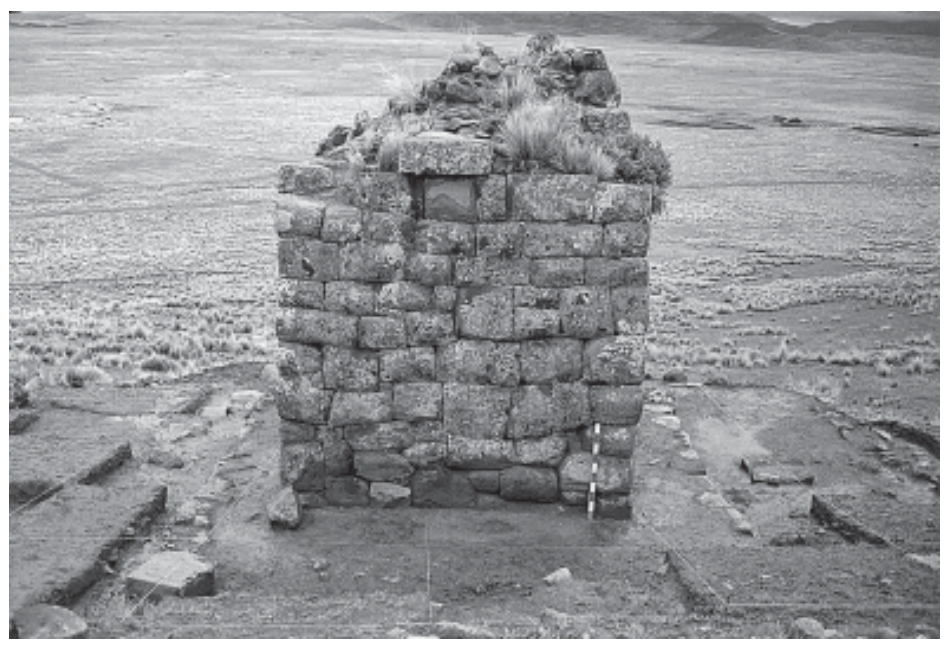

Figura 3. Vista desde el norte de la chullpa 20 durante las excavaciones. View from the North side of Chullpa 20 during excavations.

Figura 4. Vista desde el este de la chullpa 20 durante las excavaciones. Se observa la plataforma que rodea la base de la torre funeraria y frente al acceso la pequeña estructura cuadrangular utilizada para la cremación de ofrendas. View from East side Chullpa 20 during excavations. The platform that surrounds the base of the funerary lower and, in front of the access point, the small square structure used to cremate offerings, are visible.

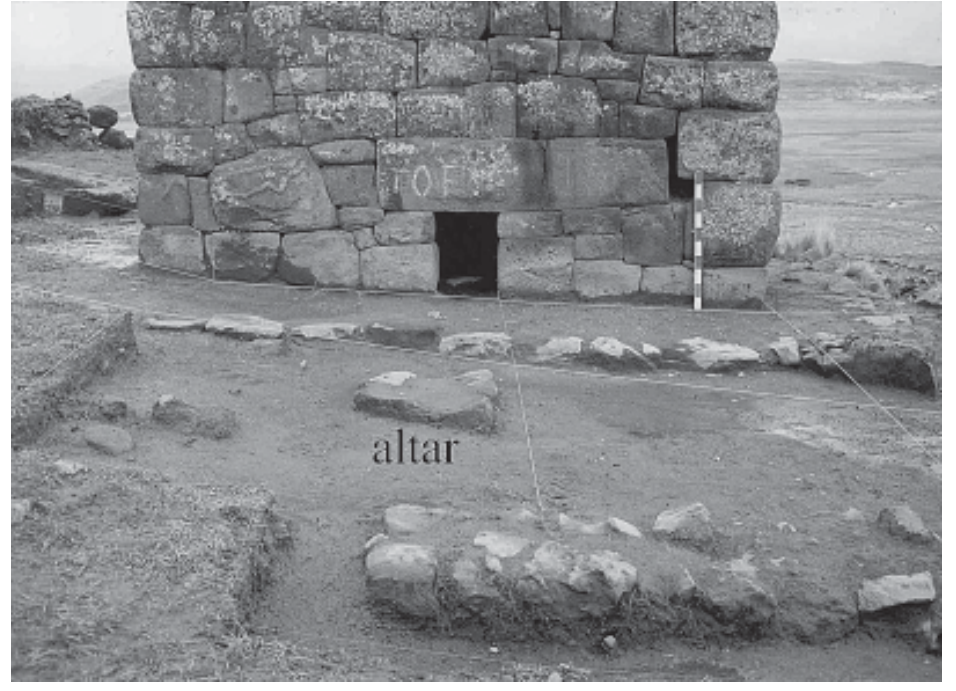

Figura 5. Vista noreste de las excavaciones en el sector principal. Se aprecian las chullpas 1 y 2 . View towards the northeast from the principal sector of excavations. Chullpas 1 and 2 are visible. 
(Figura 6). El exterior de algunos bloques de esta chullpa está decorado con diseños zoomorfos en alto relieve y representan a un roedor abundante en la zona conocido como vizcacha (Lagidium peruanum inca), a un felino probablemente un puma, un par de felinos vistos de frente y serpientes (ver ilustraciones en Hyslop 1977a). Todas estas representaciones zoomorfas son típicas en la decoración de bloques pétreos de edificios de factura inka, salvo la vizcacha, un animal que todavía habita en la meseta de Cutimbo.

La chullpa 1 es la de mayor relevancia en el sitio de Cutimbo y posiblemente una de las más importantes del altiplano circun-Titicaca (Figura 7). En la realización de la mampostería externa de esta chullpa se utilizaron dos tipos de piedra. La mayor parte está construida con bloques de andesita y las últimas hiladas superiores fueron resueltas en toba volcánica. Esta configuración de la mampostería es bastante funcional, ya que la toba volcánica absorbe el agua de lluvia impidiendo que esta fluya directamente a la base, mientras que en épocas secas su menor densidad evita la presión que se ejerce hacia la parte baja de la estructura arquitectónica, manteniendo el equilibrio total del edificio.

El interior de la cámara funeraria había sido disturbado, aunque hallamos materiales, como cerámica fina policroma con diseños Inka Imperiales e Inka asociados y una valva de Spondylus. También se rescataron entre los huesos humanos cuatro cráneos de individuos adultos con modificación artificial del tipo anular oblicuo (Blom 2005). Unas hornacinas trapezoidales, inscritas en las paredes internas norte y sur de la cámara funeraria, servirían para la colocación de las momias o mallquis. Sobre cada una de dichas hornacinas se proyectan un par de grandes ménsulas que servirían para la colocación de otros difuntos. En la pared interior oeste se inscribieron tres pequeños nichos trapezoidales, posiblemente para la colocación de ofrendas. Asimismo, la excavación del interior de las cámaras funerarias de las tres chullpas reveló que ningún cuerpo fue enterrado en el subsuelo de la cámara funeraria, por lo que éstos debieron estar expuestos en las hornacinas y ménsulas de la cámara interna de la estructura funeraria. De esta manera cumplirían su función de "sepulcros abiertos” como plantea Isbell (1997).

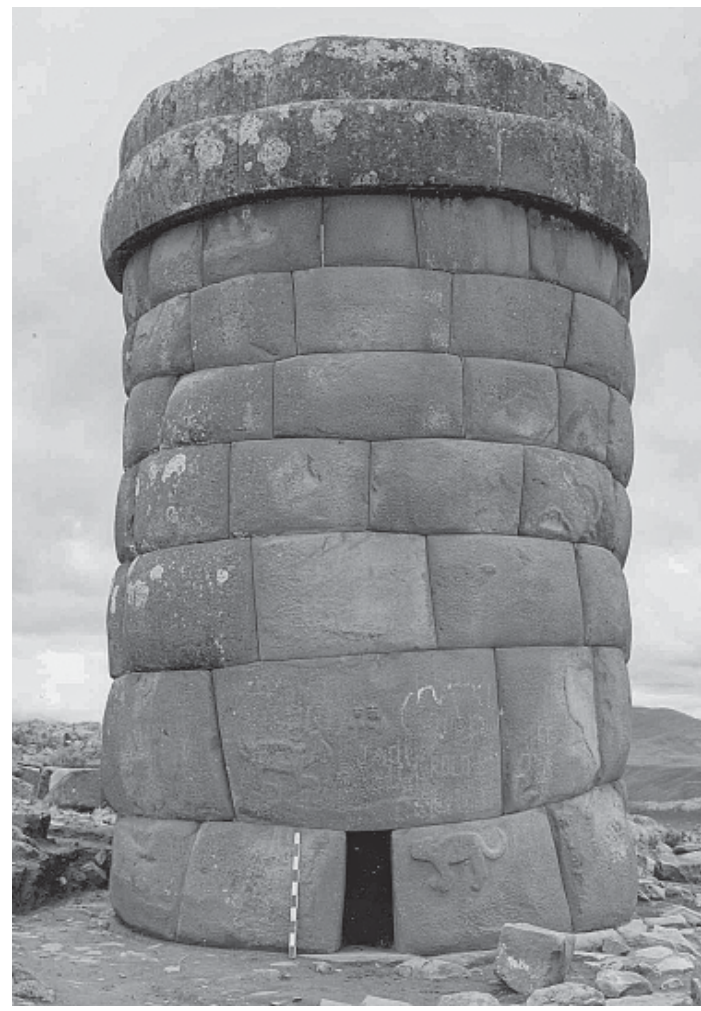

Figura 6. Chullpa 2.

Chullpa 2.

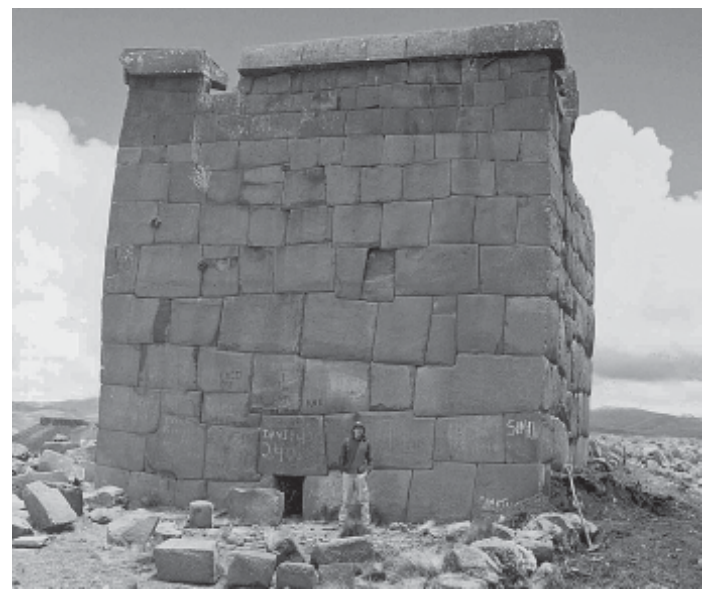

Figura 7. Chullpa 1.

Chullpa 1.

\section{Entierros en cistas asociados a las chullpas 1 y 2}

Mediante nuestra excavación en un área de 300 m cuadrados, pudimos encontrar sectores de actividad social originales en los que se localizaron tumbas de cista en torno a las chullpas, lo que su- 
ponía la contemporaneidad de ambos tipo de enterramiento y que existía un uso racional del espacio funerario, cuestión que abordaremos luego. Dos de las cistas habían sido saqueadas; sin embargo, pudimos encontrar un área original sin disturbio, que nos permitió comprender parcialmente la actividad humana prehispánica realizada en esta área del sitio donde hallamos dos cistas funerarias intactas y una estructura de piedra que contenía una urna funeraria. Dichas tumbas fueron realizadas dentro de una acumulación de material revuelto constituido por piedras y tierra (Figuras 8 y 9).

Las dos cistas halladas intactas se construyeron a cada lado de la chullpa 1 , lo que planteaba que se trataba de entierros relacionados con un ordenamiento espacial del área asociada a esta chullpa principal. También se podría barajar la posibilidad de que hayan sido sacrificios humanos, planteamiento que soporta la no-inclusión de un ajuar funerario junto al difunto, pues lo máximo que se halló en una de las cistas (cista 3) fueron dos prendedores o tupus de bronce, uno de ellos roto y reutilizado. Ambas cistas tenían paredes de piedra, forma cilíndrica y planta circular y se hallaban selladas con lajas de piedra. En cada una hallamos un esqueleto completo y articulado dispuesto en posición fetal con la parte frontal del cráneo orientada hacia al este y ambos individuos mostraban una modificación artificial de tipo anular oblicua (Blom 2005).

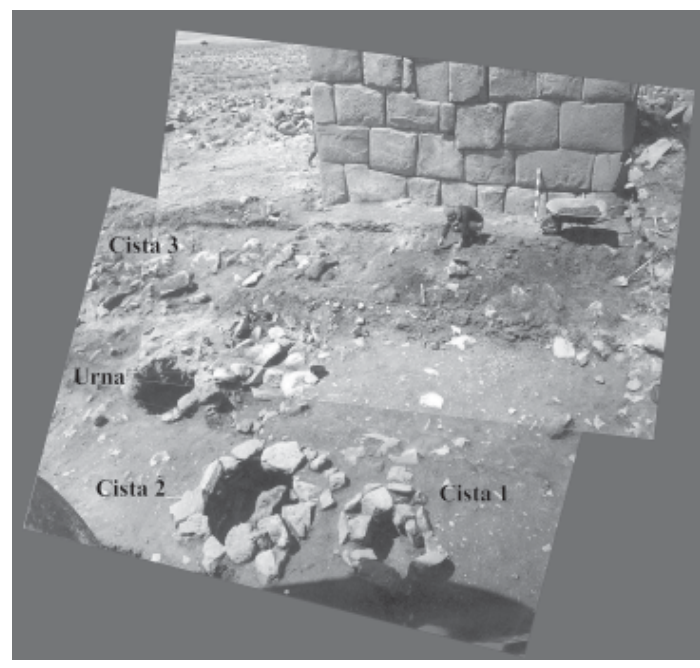

Figura 8. Area entre las chullpas 1 y 2 desde la parte superior de la chullpa 2. En primer plano las cistas 1 y 2, arriba y a la izquierda de ellas el hoyo que albergó a la urna funeraria y más arriba la cista 3 .

Area between chullpas 1 and 2 from the top portion of chullpa 2 . Cists 1 and 2 can be seen in the fore front, above and the left of them is the cavity which housed the funerary urn.

La urna funeraria. En este caso, la cobertura de la estructura funeraria estaba sellada por una capa de material arcilloso de color verdoso, que actuaba como impermeabilizante. Debajo de ésta se encontró una gran laja de piedras y dos cuñas más pequeñas a los lados, que cubrían la
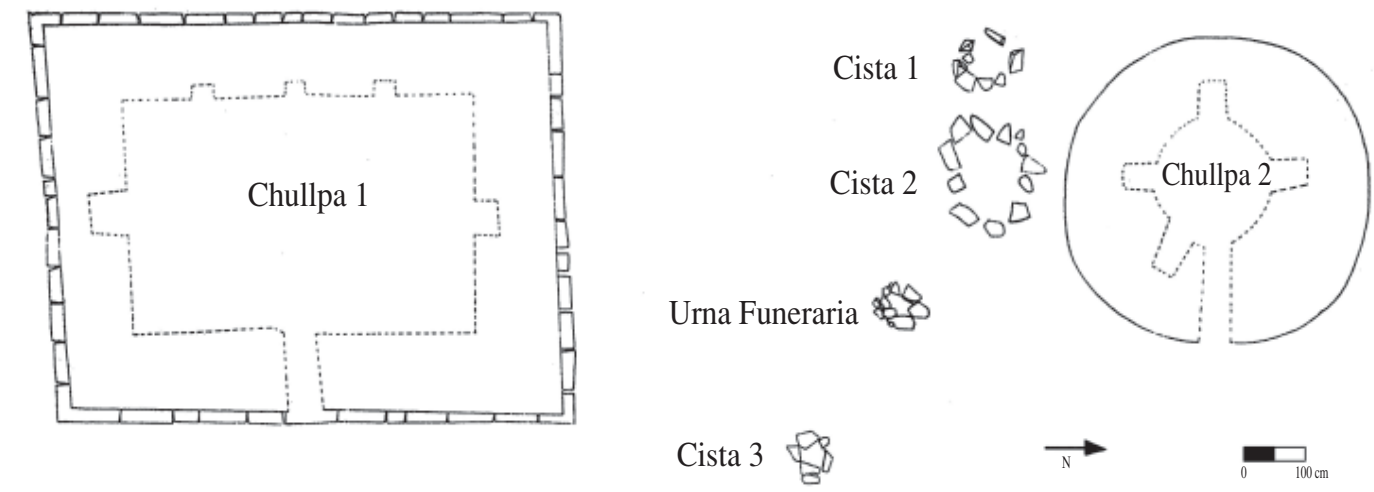

"Altar"

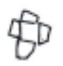

Figura 9. Planta de las excavaciones en las chullpas 1 y 2 y las principales estructuras asociadas halladas. El área de excavación excede el marco de la figura.

Plant of the excavations in chullpas 1 and 2 and of the principal associated structures. The excavation area excess the figure's frame. 
boca circular de la tumba. La relevancia de esta tumba fue su inusualidad dentro del registro arqueológico de enterramientos de época inka en el altiplano, puesto que el cuerpo se hallaba dentro de una gran vasija aribaloide o raqui (Fernández Baca 1971), utilizada generalmente para almacenar líquidos, como la chicha de maíz (Figura 10), aunque para la función que se le dio finalmente se realizaron agujeros en la base de la vasija para permitir el drenaje. La abertura de la vasija tenía un diámetro de $55 \mathrm{~cm}$, una altura de $1 \mathrm{~m}$ aproximadamente y estaba decorada con diseños geométricos en colores negro, rojo y crema. La vasija tenía las asas laterales orientadas en el eje norte-sur y el diseño principal hacia el este.

El cuerpo inhumado en la vasija correspondería al de una persona adulta (20-30 años) y aunque el cráneo se encontraba en muy mal estado de conservación, se pudo observar que presentaba modificación del tipo anular oblicuo (Blom 2005). La determinación exacta del sexo fue imposible, por la ausencia de varios huesos del esqueleto. Esto también deja abierta la posibilidad de que fuese un entierro de un cuerpo incompleto (entierro secundario) o que simplemente la mayoría de los huesos no soportasen las condiciones climáticas a lo largo del tiempo. El ajuar funerario hallado dentro de la vasija contenía: (a) un disco de bronce decorado con dos seres antropomorfos (Figura 11a), el cual pudo cumplir la función de distintivo o insignia militar, pues un objeto similar colgado del cuello de individuos plenamente reconocidos como guerreros de las milicias incaicas se observa en ilustraciones de Guamán Poma de Ayala (1987 [1615]:151, 153, 157 y 245); (b) cuatro "caleros" de bronce, los cuales representaban un instrumento de labranza conocido como chaquitaclla, sobre los que se diseñaron figuras de monos (Figura 11 b); (c) dos tupus o prendedores de bronce y (d) un anillo de plata laminada decorado con incisiones que describían rombos. Asociadas a todas estas piezas se hallaron 436 cuentas de collar confeccionadas en diversos minerales exóticos en esta área geográfica como crisocola, turquesa, lapislázuli, cristales de roca, esquisto y conchas marinas (Spondylus y Strombus). También se encontraron siete piezas de plata, tres de ellas representando ollas, otras dos vasijas aribaloides, una a un cuchillo ceremonial o tumi y otra a un animal no identificado.

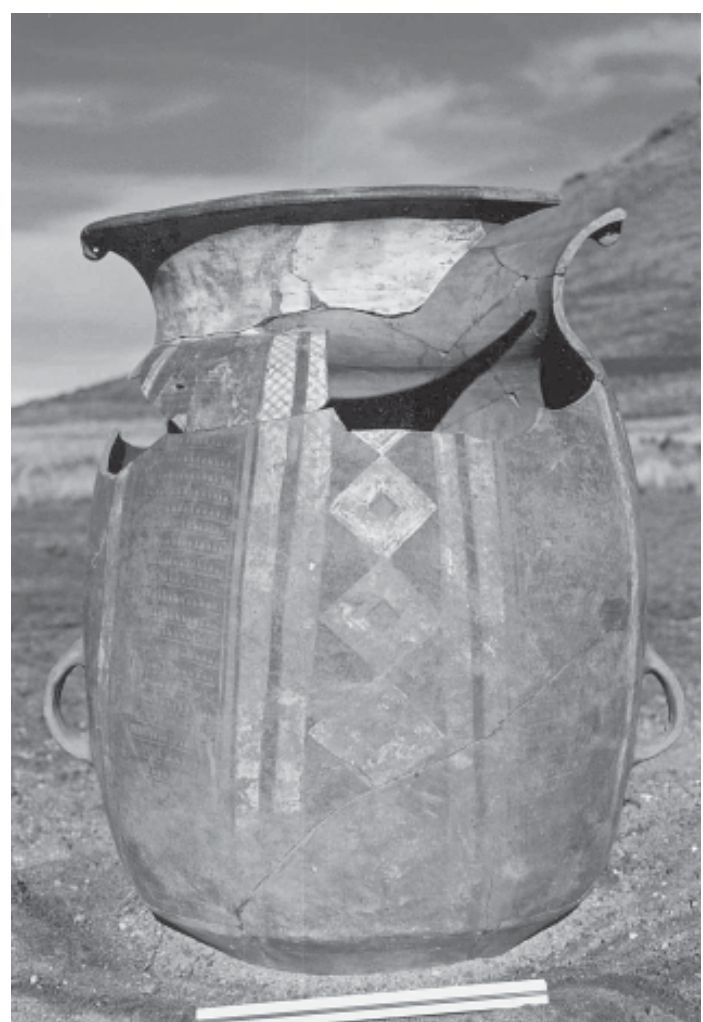

Figura 10. Vasija inka utilizada como urna funeraria, restaurada después de ser retirada de su matriz (escala $30 \mathrm{~cm}$ ).

Inka vessel used as a funerary urn, restored after being removed from matrix ( $30 \mathrm{~cm}$ scale).

Adicionalmente, a unos $20 \mathrm{~cm}$ en dirección oeste de la boca de la tumba se halló un conjunto de artefactos de metal atados con hilos de lana marrón consistente en cuatro tupus o prendedores: dos de plata, con terminación en forma de cabeza de camélido, uno con la lámina circular de oro y aguja de bronce y por último uno de plata de pequeñas dimensiones (Figura 12). A unos centímetros del anterior conjunto de artefactos se hallaron dos cuchillos ceremoniales o tumis, hechos de una aleación de cobre y estaño. El mango de uno de ellos representa de forma naturalista a un reptil que sostiene entre sus fauces a un pez (Figura 13 a), mientras que en el otro ejemplar se puede observar a un felino (Figura 13 b).

\section{Las Áreas de Cremación de Ofrendas}

Durante las excavaciones realizadas para definir las áreas de actividad originales exteriores a las chullpas 20,1 y 2 , se encontraron dos estructuras 

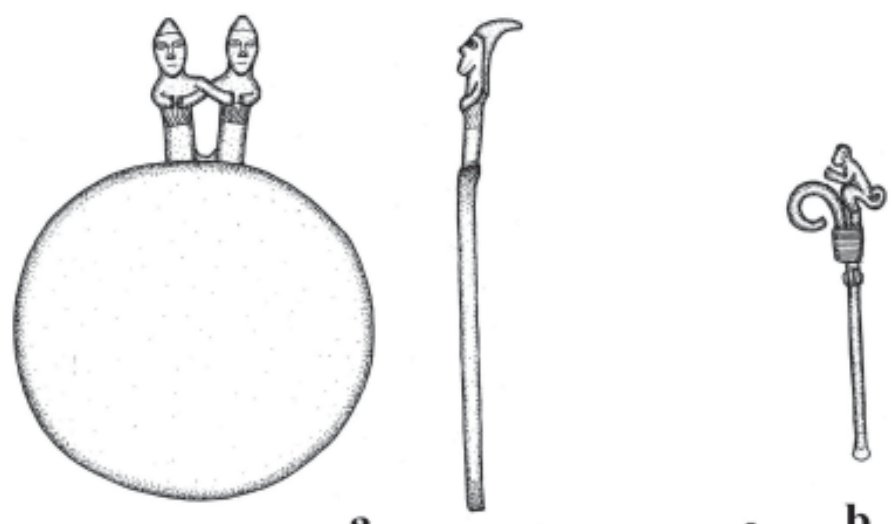

a

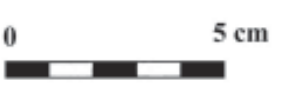

b

Figura 11. (a) Objeto circular de bronce asociado al individuo de la urna funeraria, (b) Calero de bronce recuperado en la urna funeraria.

(a) Circular bronze object associated with the individuals of the funerary urn, (b) bronze dispenser (Calero) recovered from the funerary urn.

cuadrangulares realizadas con piedras trabajadas. Alrededor de estas se halló gran cantidad de fragmentos de cerámica de alta calidad, miniaturas de vasijas cerámicas, artefactos metálicos y áreas de combustión que incluyeron huesos de camélidos y carbones vegetales.

La chullpa 20 ofreció el primer hallazgo en Cutimbo de estas áreas de actividad social externas asociadas íntimamente a la utilización original de las chullpas. El rasgo principal, en este caso, lo compuso una estructura cuadrangular $(50 \times 50 \mathrm{~cm})$ formada por piedras trabajadas que configuraban una pequeña elevación en relación con el piso que funcionó contemporáneo con la chullpa y orientada al acceso de la misma (Figura 4). Por encima y alrededor de dicha estructura se evidenció esa área de combustión antes mencionada que incluía huesos animales y carbones asociados a fragmentos de aríbalos y platos de morfología y decoración Inka imperial e Inka-Chucuito. En el caso del área exterior a la chullpa 1 , a unos dos metros de la esquina noreste, también se halló una estructura de piedras similar a la anterior (Figura 9). En el caso de la estructura de la chullpa 1, además de los elementos asociados antes mencionados para la estructura de la chullpa 20, se hallaron algunas

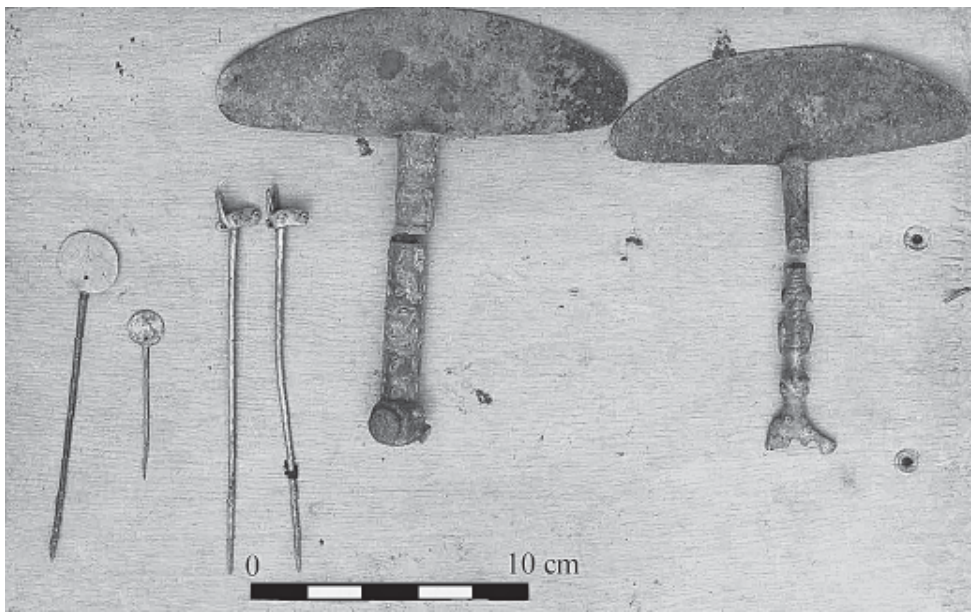

Figura 12. Artefactos encontrados cerca a la abertura de la urna funeraria. Artifacts found near the opening of the funerary urn. 


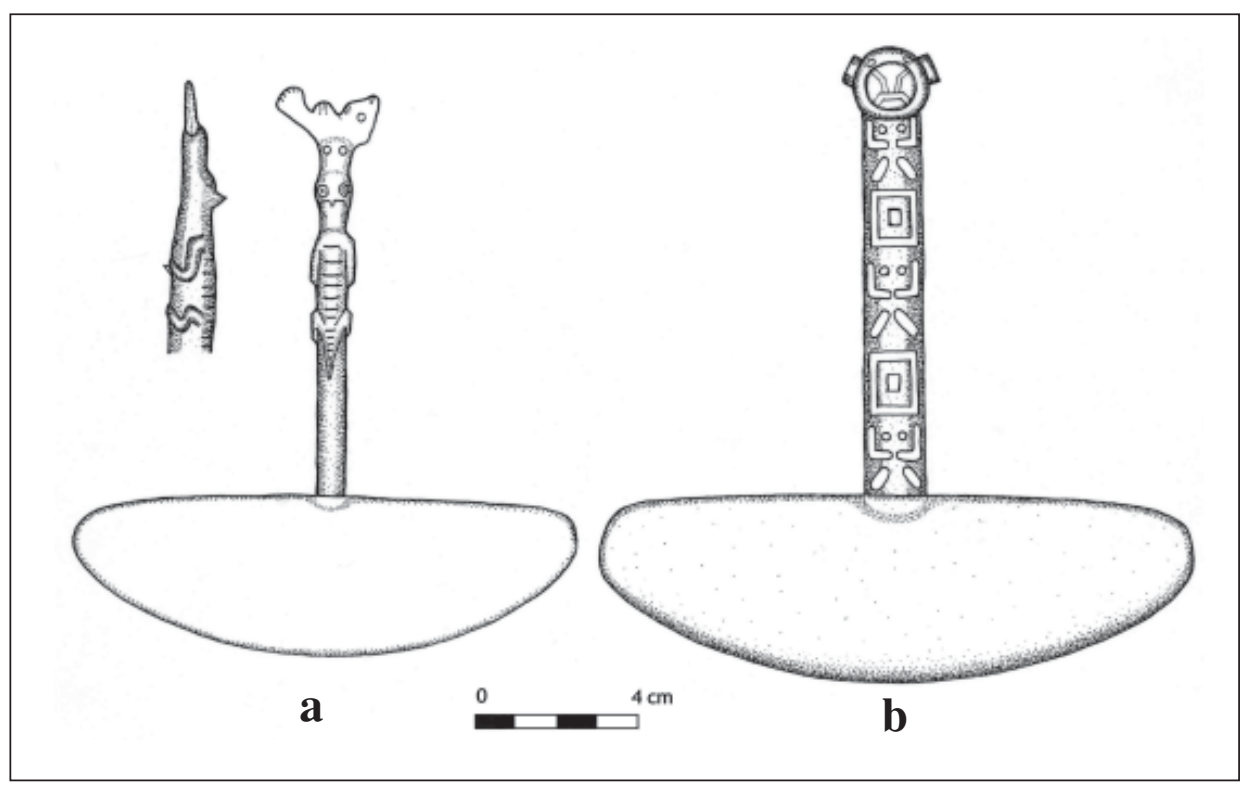

Figura 13. (a y b) Cuchillos ceremoniales hallados cerca de la abertura de la tumba de la urna funeraria. Ceremonial knives found near the opening of the tomb of the funerary urn.

piezas de metal. En ambos casos las estructuras podrían haber funcionado como "altares" o "mesas" para ofrecer simbólicamente alimentos a los ocupantes de las chullpas, como se grafica en Guamán Poma de Ayala (1987 [1615]:285, 293 y 291; ver también Isbell 1997: Figura 2.4).

También asociados directamente al área de las chullpas 1 y 2 y en su posición original debemos anotar la gran cantidad de molinos o batanes de piedra (10 solamente alrededor de las dos principales estructuras) recuperados por nuestras excavaciones, que evidenciarían la preparación de alimentos paralela a las actividades llevadas a cabo en este sector del sitio. Esta misma actividad relacionada con las chullpas monumentales ya había sido registrada con anterioridad por nosotros en el sitio de Sillustani.

\section{Discusión y Conclusiones}

La ubicación del sitio de Cutimbo en pleno altiplano, su uso anterior a la llegada de los inkas y lo inusual de las tumbas de chullpa para enterramientos de élite inka, plantearían que éstas fueron construidas para albergar los cuerpos de los señores o jefes locales lupakas (Hyslop 1977a, 1990). Además, se ha demostrado por la visita de Chucuito (Diez de San Miguel 1964 [1567]) que los curacas locales lupakas conducían la provincia a nombre del Inka (Julien 1982), condición que les proporcionaba múltiples beneficios como la concentración de riqueza, específicamente la acumulación de grandes cantidades de camélidos o el mantenimiento de su anterior jerarquía socioeconómica (Stanish 2000), lo que significaría el mantenimiento de la explotación del trabajo de miembros de la misma comunidad y que mantendría categorías socioeconómicas como urus o "indios pobres" y que compondrían la fuerza de trabajo necesaria para la construcción de obras como las chullpas funerarias monumentales.

De hecho, las estructuras funerarias monumentales no serían la única muestra de arquitectura con rasgos inka construidas por los lupaka en Cutimbo, pues existe todo un sector al noreste de las chullpas 1 y 2 compuesto por edificios de planta rectangular con características inka pero indudablemente construidas por arquitectos y mano de obra locales. Adicionalmente, el mismo Inka, Uyu de la ciudad de Chucuito, fundada durante la ocupación inka, habría sido construida por arquitectos locales (Arkush 2005:236). Si bien la utilización de una arquitectura funeraria similar fue utilizada durante la ocupación inka en los territorios colla y pakaje, la elite lupaka, que es la mejor candidata para la construcción y utilización de las 
chullpas monumentales de Cutimbo, conservaría un poder socioeconómico tal que le posibilitaría realizar dichas construcciones funerarias, además en un lugar de (re)encuentro social relacionado con su autonomía local, bastante apartado de las rutas oficiales de la religión inka (Arkush 2005) e incluso alejado y aislado de los asentamientos humanos fundados durante la ocupación inka ${ }^{6}$.

Por otro lado, las cuevas funerarias, las cistas y sobre todo la tumba de la urna funeraria como forma de enterramiento diferentes a las chullpas en Cutimbo representaría que los lupakas, aunque inkaizados, seguían manteniendo sus propias asimetrías socioeconómicas. Esto trascendería en el momento de la muerte de un miembro de la elite lupaka de menor rango, enterrándolo en un área periférica a una estructura mayor de elite, no pudiendo ingresar su cuerpo en dicho edificio, por no pertenecer al grupo social privilegiado. Asimismo, la urna funeraria pertenecería a un individuo relacionado con las actividades militares, como demuestra el objeto hallado en la tumba semejante al de las representaciones de Guamán Poma de Ayala. Adicionalmente, el depósito o amortización de artefactos relacionados a la tumba expresarían su pertenencia a un grupo socioeconómico de mayor jerarquía dentro de la sociedad lupaka-inka ${ }^{7}$.

Con relación a los hallazgos al exterior y frente a las principales estructuras funerarias del sitio, sabemos por referencias etnohistóricas que alrededor de las chullpas se congregaban grupos de personas periódicamente para realizar fiestas y rituales (materializados en las trazas del consumo y amortización de alimentos y artefactos) dirigidos a los ocupantes de las estructuras funerarias, como, por ejemplo, señala Guamán Poma de Ayala (1987 [1614]). Asimismo, dicha representación de las actividades sociales, en este caso rituales relacionados con los muertos depositados en las estructuras funerarias, encaja bien con la propuesta de los "sepulcros abiertos" de Isbell (1997). Aunque el planteamiento de este autor trata de relacionar directamente a dichas estructuras funerarias con el tema de la reproducción de los ayllus, nosotros planteamos que complementariamente a esa construcción de los ayllus (al fin y al cabo, una ideología cohesionadora de producción de identidad colectiva), aquí el objetivo real sería la reproducción sociopolítica de las elites locales lupaka durante un período de dominación externa, en este caso inka.
De este modo, Cutimbo incluyó lugares sociales donde se realizaron prácticas sociopolíticas de forma periódica relacionadas con los muertos a los que se trataba como en vida. Los diferentes tipos de prácticas funerarias de una misma época hallados aquí reflejan la importancia del yacimiento en el ámbito religioso y político, poniendo en evidencia sobre todo las diferencias sociales que existían en ese momento.

Asimismo, el proceso de enriquecimiento y retención de poder económico y político de la elite lupaka al que se habían arriesgado los dirigentes inka, originó en algún momento la debilitación del orden impuesto y llevado a disputas entre la elite local y la elite inka (como hacen referencia las fuentes etnohistóricas). Aun cuando se evidencia un fuerte control ideológico materializado en diferentes espacios rituales (Arkush 2005), lugares como Cutimbo sirvieron para evadir el espacio de coerción ideológica impuesto por los inkas, sirviendo para que la elite lupaka recreara las distancias sociales y la ideología que las justificaba. Para ello periódicamente los lupaka regresaron a Cutimbo para construir, además de sus chullpas y otras estructuras funerarias, su ideología y así conservar sus jerarquías socioeconómicas propias y su conciencia de grupo social con relación a los inkas.

Todo esto nos conduce a plantearnos que las estrategias de dominación inka no siempre fueron tan efectivas (aunque sirviesen para sus objetivos primordiales) y como mediante ciertas prácticas sociales las poblaciones locales resistieron durante mucho tiempo las ocupaciones externas, un proceso dialéctico que se evidencia hasta bien entrado el siglo XVI.

Agradecimientos: Este artículo nunca podría haber sido escrito sin la confianza que depositó en mí Rolando Paredes Eyzaguirre, director del Instituto Nacional de Cultura-Puno. También deseo agradecer a Carmen Pérez Maestro, incomparable compañera en la fría puna durante las campañas de excavación; a Eduardo Arizaca, arqueólogo ayavireño, de quien aprendimos los conocimientos empíricos de la zona y al equipo de trabajo que conformamos con los pobladores de la zona: Roy Marón, Sixto Quispe y Abdón Chambilla. A Charles Stanish por su constante apoyo y sus acertados comentarios a este manuscrito. También agradezco a los dos consultores externos por sus críticas que me ayudaron a enfocar y organizar mi texto. 
Muchas de mis ideas acerca del "mundo de los muertos" cambiaron sustancialmente en la Universidad Autónoma de Barcelona, por eso, muchas de las ideas aquí vertidas son consecuencia de las discusiones en el Departamento de Prehistoria de dicha Universidad aunque quisiera agradecer espe- cialmente a Vicente Lull quien ha sido una gran inspiración para mí. Marillyn Holmes ocupa un importante espacio en mi vida y gracias a ella puedo sentirme realizado en muchos aspectos. Las ilustraciones que acompañan al texto fueron mejoradas por Juan Roel.

\section{Referencias Citadas}

Arkush, E.

2005 Inka Ceremonial sites in the Southwest Titicaca Basin. En Advances in Titicaca Basin Archaeology-1, editado por C. Stanish, A.B. Cohen y M. Aldenderfer, pp. 209-242. Cotsen Institute of Archaeology, Los Angeles.

Ayca, O.

1995 Sillustani. Instituto de Arqueología del Sur, Tacna.

Bandelier, A.

1905 The aboriginal ruins at Sillustani, Perú. American Anthropologist 7:49-68.

Bauer, B. y C. Stanish

2001 Ritual and Pilgrimage in the Ancient Andes. The Islands of the Sun and the Moon. University of Texas Press, Austin.

Blom, D.

2005 Tiwanaku group dynamics: A bioarchaeological approach. En Us and Them: Archaeology and Ethnicity in the Andes, editado por R. Reycraft, pp. 153-182. Contribution of the University of California Archaeological Research Facility, Los Angeles.

Castro, P., R. Chapman, S. Gili, V. Lull, R. Micó, C. Rihuete, R. Risch y M.E. Sanahuja

1996 Teoría de las prácticas sociales. Complutum 6:35-48.

Castro, P., S. Gili, V. Lull, R. Micó, C. Rihuete, R. Risch y M.E. Sanahuja

1998 Teoría de la producción de la vida social. Mecanismos de explotación en el sudeste Ibérico. Boletín de Antropología Americana 33:25-77.

Cieza de León, P.

1929[1553] La Crónica General del Perú. Colección Urteaga. Historiadores Clásicos del Perú. Imprenta Gil, Lima.

Cobo, B.

1964[1653] Historia del Nuevo Mundo. Biblioteca de Autores Españoles, 91-92. Ediciones Atlas, Madrid.

de la Vega, E., K. Frye y T. Tung

2005 The Cave Burial from Molino Chilacachi. En Advances in Titicaca Basin Archaeology-1, editado por C. Stanish, A B. Cohen y M. Aldenderfer, pp. 185-196. Cotsen Institute of Archaeology, Los Angeles.

Diez de San Miguel, G.

1964[1567] Visita Hecha a la Provincia de Chucuito por Garci Diez de San Miguel en 1567. Documentos Regionales para la Etnología y Etnohistoria Andina 1:1-287. Casa de la Cultura, Lima.

Fernández Baca, J.

1971 Motivos de Ornamentación Inca-Cuzco. Tomo I y II. Librería Studium Ed., Lima.
Frye, K.

1997 Political centralization in the Altiplano Period in the southwestern Titicaca Basin. En Archaeological Survey in the Juli-Desaguadero Region of Lake Titicaca Basin, Southern Peru, editado por C. Stanish, E. de la Vega, L. Steadman, C. Chávez, K. Frye, L. Mamani, M. Seddon y P. Chuquimia, pp. 129-141. Fieldiana 29, Chicago.

2005 The Inca Occupation of the Lake Titcaca Region. En Advances in Titicaca Basin Archaeology-1, editado por C. Stanish, A.B. Cohen y M. Aldenderfer, pp. 197-208. Cotsen Institute of Archaeology, Los Angeles.

Frye, K. y E. de la Vega

2005 The Altiplano Period in the Titicaca Basin. En Advances in Titicaca Basin Archaeology-1, editado por C. Stanish, A.B. Cohen y M. Aldenderfer, pp. 173-184. Cotsen Institute of Archaeology, Los Angeles.

Gil García, F.

2000 Manejo Simbólico del Espacio Andino y Estructuras Chullparias. Propuestas Interpretativas. Tesis de Licenciatura, Departamento de Historia de América II (Antropología de América), Universidad Complutense de Madrid.

2001 De "tumbas reales" a "chullpas-en-el-paisaje" pasando por los "ayllus de sepulcros abiertos". Reflexiones epistemológicas sobre casi dos siglos de arqueología del fenómeno chullpario. http://www.ucm.es/info/arqueoweb/ numero3_3/articulo3_3_gil.html (diciembre 2001).

Gisbert, T., J.C. Jemio y R. Montero

1996 El señorío de los Caranga y los chulpares del río Lauca. Revista de la Academia Nacional de Ciencias de Bolivia 70:3-66.

Guamán Poma de Ayala, F.

1987[1615] Primer Nueva Crónica y Buen Gobierno, editado por J. Murra, R. Adorno y J.L. Uriaste. Historia 16, Madrid.

Gutiérrez Flores, P.

1964[1574] Padrón de los Mil Indios Ricos de la Provincia de Chucuito. En Diez de San Miguel [1567] 1964:305-306. Documentos Regionales para la Etnología y Etnohistoria Andina 1. Casa de la Cultura, Lima.

Hyslop, J.

1976 An Archaeological Survey of the Lupaqa Kingdom and its Origins. Ph.D. dissertation, Department of Anthropology, Columbia University.

1977a Chulpas of the Lupaca zone of the Peruvian high plateau. Journal of Field Archaeology 4:149-170. 
1977b Hilltop cities in Peru. Archaeology 30:218-226.

1978 El Área Lupaca bajo el dominio incaico: un reconocimiento arqueológico. Revista Histórica III(1):53-73.

1984 The Inka Road System. Academic Press, New York. 1990 Inka Settlement Planning. University of Texas Press, Austin.

Isbell, W.

1997 Mummies and Mortuary Monuments. A Posprocessual Prehistory of Central Andean Social Organization. University of Texas Press, Austin.

Julien, C.

1982 Inka Decimal Administration in the Lake Titicaca Region. En The Inca and Aztec States, 1400-1800: Anthropology and History, editado por G.A. Collier, R.I. Rosado y J.D. Wirth, pp. 119-151. Academic Press, New York and London.

1983 Hatunqolla: A view of Inka rule from the Lake Titicaca Region. Series Publications in Anthropology № 15 . University of California Press, Berkeley.

Kesseli, R.

2001 The grave towers of the Bolivian high plateau. En The Incas and their Predecessors. Three Millennia of Precolumbian Peru, editado por A. Ilmonen y J. Talvitie, pp. 352355. Tampere Art Museum, Tampere.

Kesseli, R., P. Lihua, M. Rossi y J. Bustamante

1999 Archaeological and geographical research of precolumbian (AD 1200-1532) grave towers or chulpa on the Bolivian high plateau in the years 1989-1998. Preliminary report. En Dig it All. Papers Dedicated to Ari Siriiäinen, editado por M. Huurre, pp. 335-348. The Finish Antiquarian Society-The Archaeological Society of Finland, Helsinki.

Kolata, A.

1993 The Tiwanaku: Portrait of an Andean Civilization. Blackwell, Cambridge.

Lull, V.

2000 Death and society: A Marxist Approach. Antiquity 74:576-580.

Lumbreras, L.G.

1974 Los reinos post-Tiwanaku en el área altiplánica. Revista del Museo Nacional 40:55-85.

Malpass, $\mathrm{M}$.

1993 Provincial Inca. Archaeological and Ethnohistorical Assessment of the Impact of the Inca State. University of Iowa Press, Iowa.

Murra, J.

1968 An aymara kingdom in 1567. Ethnohistory XV: 115-151.
Nordenskiöld, E.

1953 Investigaciones Arqueológicas en la Región Fronteriza de Perú y Bolivia. Biblioteca Paceña, La Paz.

Squier, E.

1974[1877] Un Viaje por Tierras Incaicas. Crónica de una Expedición Arqueológica (1863-1865). Amigos del Libro, La Paz.

Stanish, C.

1992 Ancient Andean Political Economy. University of Texas Press, Austin.

1993 The hydraulic hypothesis revisited: Lake Titicaca Basin raised fields in theoretical perspective. Latin American Antiquity 5:312-332.

2000 Negotiating rank in an Imperial State: Lake Titicaca Basin elite under Inca and spanish control. En Hierarchies in Action: cui Bono?, editado por M. Diehl, pp. 317-339. Southern Illinois University Center for Archaeological Investigations. Ocassional Paper $\mathrm{N}^{\circ} 27$, Carbondale.

2001 Regional research on the Inca. Journal of Archaeological Research 9:213-241.

2003 Ancient Titicaca: The Evolution of Complex Society in Southern Peru and Northern Bolivia. University of California Press, Berkeley and Los Angeles.

Stanish, C., A. Cohen y M. Aldenderfer, editores

2005 Advances in Titicaca Basin Archaeology-1. Cotsen Institute of Archaeology. University of California of Los Angeles, Los Angeles.

Stanish, C., E. de la Vega, L. Steadman, C. Chávez., K.L. Frye,

L. Mamani, M. Seddon y P. Chuquimia

1997 Archaeological survey in the Juli-Desaguadero Region of Lake Titicaca Basin, Southern Peru. Fieldiana Anthropology 29.

Tantaleán, H. y C. Pérez Maestro

2000 Muerte en el Altiplano Andino: Investigaciones en la Necrópolis Inka de Cutimbo (Puno, Perú). Revista de Arqueología 228:26-37.

Tschopik, M.H.

1946 Some notes of the archaeology of the Department of Puno. Peabody Museum of American Archaeology and Ethnology Papers 27(3), Harvard University, Cambridge.

Vázquez, E.

1937 Sillustani: Una metrópoli pre-incásica. Revista del Museo Nacional 6:278-290.

1939 Ruinas Arqueológicas de Puno, Qutimpu. Revista del Museo Nacional 8:117-123.

Yaeger, J y J.M. Bejarano

2004 Reconfiguración de un espacio sagrado: los Inkas y la pirámide Pumapunku en Tiwanaku, Bolivia. Chungara, Revista de Antropología Chilena 36:337-350.

\section{Notas}

1 Nuestra perspectiva teórica materialista dialéctica está inspirada en la teoría de las prácticas sociales (Castro et al. 1996) y la teoría de la producción de la vida social (Castro et al. 1998). Para el caso de los inka, aproximaciones similares que inspiran este planteamiento son sintetizadas en Stanish 2001:220-221.

2 Otro motivo para la expansión de los inka hacia el altiplano del Titicaca sería el ideológico patentizado en la ocupación de Tiwanaku (Kolata 1993:1-10; Yaeger y Bejarano 2004) o de las islas del Sol y la Luna (Bauer y Stanish 2001).

3 En relación a esto, el tema de los urus o pobres en el área lupaka (Stanish 2000) es relevante para la aceptación de grupos sociales locales explotados antes, durante e incluso después de la ocupación Inka.

4 Habría que determinar si realmente las provincias inka corresponden a los territorios originales que ocupaban 
las sociedades en el período Altiplano (1.100 d.C.1.470 d.C.).

5 Hay que precisar que las excavaciones en Cutimbo se realizaron como parte de un proyecto realizado por un convenio entre el Gobierno Regional de Puno y el Instituto Nacional de Cultura durante los meses de febrero y marzo de 1998 con el objetivo principal de realizar una "puesta en valor" del sitio. A pesar que el objetivo principal era restaurar las chullpas monumentales y acondicionar una vía de acceso a las mismas, pudimos sacarle provecho a dicho proyecto creando una estrategia de investigación que optimice la información generada a raíz de esta oportunidad única para explorar e investigar las chullpas monumentales y sus áreas asociadas. Bajo esta misma premisa también encargamos a la arqueóloga Carmen Pérez Maestro la prospección de los abrigos rocosos y cuevas de Cutimbo y que incluyó el relevamiento del arte rupestre allí plasmado. Eduardo Arizaca, además de excavar junto a nosotros el área de las chullpas monumentales, excavó algunas estructuras circulares para deslindar su función.

6 Aun con todo, cabría la posibilidad que las tumbas perteneciesen a la elite cuzqueña, a la espera de un estudio que contraste el estudio del "mundo de los muertos" (espacios funerarios) con el "mundo de los vivos" (espacios de producción de la vida social) (Lull 2000) en Cutimbo y alrededores.

7 Sin embargo, la inhumación en la urna podría ser resultado de un sacrificio, entrando así en otro tipo de práctica sociopolítica también relacionada con la reproducción de las asimetrías sociales. 
